\title{
Usefulness of speckle tracking echocardiography and biomarkers for detecting acute cellular rejection after heart transplantation
}

Cecilia Beatriz Bittencourt Viana Cruz ${ }^{1,2^{*}}$, Ludhmila A. Hajjarr ${ }^{1}$, Fernando Bacal ${ }^{1}$, Marco S. Lofrano-Alves ${ }^{1}$, Márcio S. M. Lima ${ }^{1,2}$, Maria C. Abduch", Marcelo L. C. Vieira', Hsu P. Chiang ${ }^{1,2}$, Juliana B. C. Salviano ${ }^{1}$, Isabela Bispo Santos da Silva Costa', Julia Tizue Fukushima', Joao C. N. Sbano ${ }^{1,2}$, Wilson Mathias Jr ${ }^{1,2}$ and Jeane M. Tsutsui ${ }^{1,2}$

\begin{abstract}
Background: Acute cellular rejection (ACR) is a major complication after heart transplantation. Endomyocardial biopsy (EMB) remains the gold standard for its diagnosis, but it has concerning complications. We evaluated the usefulness of speckle tracking echocardiography (STE) and biomarkers for detecting ACR after heart transplantation.

Methods: We prospectively studied 60 transplant patients with normal left and right ventricular systolic function who underwent EMB for surveillance 6 months after transplantation. Sixty age- and sex-matched healthy individuals constituted the control group. Conventional echocardiographic parameters, left ventricular global longitudinal, radial and circumferential strain (LV-GLS, LV-GRS and LV-GCS, respectively), left ventricular systolic twist (LV-twist) and right ventricular free wall longitudinal strain (RV-FWLS) were analyzed just before the procedure. We also measured biomarkers at the same moment.

Results: Among the 60 studied patients, 17 (28\%) had severe ACR (grade $\geq 2 R$ ), and 43 (72\%) had no significant ACR (grade $0-1 R$ ). The absolute values of LV-GLS, LV-twist and RV-FWLS were lower in transplant patients with ACR degree $\geq 2 R$ than in those without ACR $\left(12.5 \% \pm 2.9 \%\right.$ vs $14.8 \% \pm 2.3 \%, p=0.002 ; 13.9^{\circ} \pm 4.8^{\circ}$ vs $17.1^{\circ} \pm 3.2^{\circ}$, $p=0.048 ; 16.6 \% \pm 2.9 \%$ vs $21.4 \% \pm 3.2 \%, p<0.001$; respectively), while no differences were observed between the LV-GRS or LV-GCS. All of these parameters were lower in the transplant group without ACR than in the nontransplant control group, except for the LV-twist. Cardiac troponin I levels were significantly higher in patients with significant ACR than in patients without significant ACR $[0.19 \mathrm{ng} / \mathrm{mL}(0.09-1.31) \mathrm{vs} 0.05 \mathrm{ng} / \mathrm{mL}(0.01-0.18), p=$ 0.007]. The combination of troponin with LV-GLS, RV-FWLS and LV-Twist had an area under curve for the detection of ACR of 0.80 (0.68-0.92), 0.89 (0.81-0.93) and 0.79 (0.66-0.92), respectively.

(Continued on next page)
\end{abstract}

\footnotetext{
* Correspondence: cbbvc@yahoo.com.br

${ }^{1}$ Heart Institute (InCor), University of São Paulo Medical School, Av. Dr. Enéas de Carvalho Aguiar, 44, São Paulo, SP 05403-000, Brazil

Fleury Medicine \& Health, São Paulo, Brazil
}

(c) The Author(s). 2021, corrected publication January 2021. Open Access This article is licensed under a Creative Commons Attribution 4.0 International License, which permits use, sharing, adaptation, distribution and reproduction in any medium or format, as long as you give appropriate credit to the original author(s) and the source, provide a link to the Creative Commons licence, and indicate if changes were made. The images or other third party material in this article are included in the article's Creative Commons licence, unless indicated otherwise in a credit line to the material. If material is not included in the article's Creative Commons licence and your intended use is not permitted by statutory regulation or exceeds the permitted use, you will need to obtain permission directly from the copyright holder. To view a copy of this licence, visit http://creativecommons.org/licenses/by/4.0/. The Creative Commons Public Domain Dedication waiver (http:// creativecommons.org/publicdomain/zero/1.0/) applies to the data made available in this article, unless otherwise stated in a credit line to the data. 
(Continued from previous page)

Conclusion: Heart transplant patients have altered left ventricular dynamics compared with control individuals. The combination of troponin with strain parameters had higher accuracy for the detection of ACR than the isolated variables and this association might select patients with a higher risk for ACR who will benefit from an EMB procedure in the first year after heart transplantation.

Keywords: Heart transplantation, Acute cellular rejection, Speckle tracking echocardiography

\section{Introduction}

Heart transplantation is the treatment of choice for selected patients with end-stage heart failure [1, 2]. Although significant advances in immunosuppressive therapy have been beneficial in decreasing cardiac allograft rejection, graft failure remains one of the major associated complication [3, 4]. For this reason, adequate monitoring of heart transplant patients to diagnose and initiate specific therapy for transplant rejection in a timely manner is important, albeit challenging.

Endomyocardial biopsy (EMB) is the widely accepted gold standard for the diagnosis of acute cellular rejection (ACR) [5]. However, it is invasive and is associated with complications in 0.2 to $5.5 \%$ of cases; these complications include tricuspid regurgitation, cardiac perforation and cardiac tamponade [6-9]. Additional limitations of EMB include the subjectivity of the pathological analysis, and significant interobserver variability, which compromises its reliability and reproducibility [10-12].

Therefore, there has been considerable effort exerted to develop noninvasive and accurate methods that can reduce the need for EMB, including biomarker detection, imaging techniques and genetic tests $[13,14]$. The most frequently used biomarkers in patients with heart transplantation are troponin and B-type natriuretic peptide (BNP). However, there is considerable heterogeneity among studies about the timing of their use and their predictive value for detecting ACR $[15,16]$.Two-dimensional speckle-tracking echocardiography (STE) is an echocardiographic modality for the evaluation of myocardial deformation, enabling the determination of multiple parameters involved in cardiac mechanics, and it has emerged as a promising tool for detecting early subclinical cardiac dysfunction in many different scenarios $[17,18]$. Previous studies in the heart transplant population demonstrated that, in this setting, the data are not definitive, and further exploration of cardiac mechanics is necessary $[19,20]$.

In the present study, we aimed to assess the value of STE-derived strain measurements and biomarkers for the noninvasive detection of ACR after heart transplantation. We also evaluated the ventricular dynamics of heart transplant patients in comparison with control individuals using STE.

\section{Methods}

\section{Patients}

From January 2014 to November 2018, we prospectively studied heart transplant patients who underwent EMB in the 6th month after orthotopic heart transplantation for the diagnosis of ACR during routine surveillance. All of the included patients were asymptomatic and did not present with any hemodynamic compromise at the time of enrollment.

The exclusion criteria were as follows: age $<18$ years, arrhythmia, left ventricular ejection fraction $<0.55$, right ventricular dysfunction of any degree (fractional area change $<35 \%$ ), vascular graft disease, humoral rejection, two or more previous cellular rejection episodes, chronic kidney disease, Chagas disease reactivation, limited echocardiographic window for STE analysis and an inconclusive EMB analysis.

All patients underwent the same protocol according to the predefined steps. First, enrolled patients underwent a complete echocardiographic analysis for the assessment of left and right ventricular function. For those with a normal systolic function, images were acquired for mechanical analysis by STE, and blood was taken for biomarker tests. The patients then underwent EMB and, according to the results, were divided into the following groups: 1) without significant ACR (grades 0 and $1 \mathrm{R}$ ) and 2) with significant ACR (grades $2 R$ and $3 R$ ).

Individuals without a clinical history of any disease known to interfere with myocardial physiology or structure and who were matched by sex and age with the transplant patients constituted the control group. This group underwent STE for the analysis of ventricular mechanics and was compared to the groups of transplant patients with and without significant ACR. The study protocol was approved by our ethical committee, and all patients gave written informed consent to participate.

\section{Endomyocardial biopsy}

EMB was performed through the internal jugular or femoral vein under radioscopy. A minimum of 3 distinct ventricular myocardial fragments were collected (each consisting of at least $50 \%$ myocardium) and sent for anatomopathological analysis. A sample was considered sufficient when at least 3 myocardial fragments were obtained for analysis by optical microscopy after fixation 
in $10 \%$ formalin and staining of the laminae with hematoxylin and eosin. Two experienced cardiac pathologists blinded to the echocardiographic results analyzed all biopsies. The grade of rejection was based upon the recommendations of the International Society for Heart and Lung Transplantation [21]. The results of the EMB were described as grade 0 (without rejection), grade $1 R$ (mild rejection, low grade), grade $2 \mathrm{R}$ (moderate rejection, intermediate grade) or grade $3 R$ (severe rejection, high grade) [21]. In our study, grades $2 \mathrm{R}$ and $3 \mathrm{R}$ were considered as significant ACR.

\section{Echocardiography}

On the day of EMB, just before the procedure, patients underwent echocardiographic examination on a commercially available machine equipped with an MS5 probe (GE Vivid 9, GE Healthcare, Milwaukee, Wisconsin, USA). Image acquisition and assessment were performed according to the recommendations of the American Society of Echocardiography [22]. Left ventricular ejection fraction was obtained by Simpson's rule throughout apical 4- and 2-chamber views, and the left ventricular mass was calculated using the equation proposed by Devereux et al. [23], indexed by body surface area to derive the left ventricular mass index. Right ventricular systolic function was assessed with the conventional parameters recommended for routine clinical practice: tricuspid annular plane systolic excursion, systolic excursion velocity, and fractional area change, which were obtained with M-mode, pulsed tissue Doppler and two-dimensional echocardiography, respectively. Diastolic function was evaluated based on mitral inflow E/A pattern, E/A ratio, E velocity deceleration time, annular tissue Doppler curves (e'/a'), and E/e' ratio.

To assess the ventricular mechanics, 3 consecutive cardiac cycles were recorded. Left ventricular short-axis and apical views were acquired using two-dimensional grayscale second-harmonic imaging at a frame rate of 50-80 frames per second. Left ventricular short-axis views at the basal, mid (papillary muscles) and apex levels were acquired to analyze the circumferential (LVGCS) and radial strain (LV-GRS), while the left ventricular apical 4-, 2- and 3-chamber views were used to assess the left ventricular global longitudinal strain (LV-GLS) and the apical 4-chamber view focused on the right ventricle was used to analyze the right ventricular free wall longitudinal strain (RV-FWLS).

STE analysis was performed offline using dedicated software (EchoPAC, version BT11, GE Healthcare). All echocardiographic measurements were performed by one specialist blinded to the clinical data. End-systole was determined by pulsed-wave Doppler at the time of aortic valve closure. After the ventricular endocardial border was manually traced, the epicardial borders were automatically defined to create regions of interest according to the ventricular segmentation; if necessary, adjustments were made by the operator. In particular, care was taken to not include the myocardial trabeculae and the pericardium. Following this step, the myocardial speckles were automatically tracked by the dedicated software, and, in the case of suboptimal tracking, further manual adjustments were allowed, resulting in strain curves that were exported to a spreadsheet. Global longitudinal strain, circumferential strain, and radial strain were calculated as averages of peak systolic strain values obtained from all segments in the respective views. Accordingly, the LV-GLS was obtained from the mean of 18 segments acquired in the apical 4-, 2-, and 3chamber views, while circumferential and radial strain were obtained from 12 segments in LV short-axis views at the basal, papillary muscle levels and apex. Left ventricular twist (LV-Twist) is the wringing motion of the heart around its long axis. It was calculated as the net absolute difference between the apical and basal rotations (LV-Twist $=$ ROTapical - ROTbasal). By widely assumed convention, apical rotation has positive values and basal had negative values [24]. The RV-FWLS was obtained by averaging the values of the 3 right ventricular free wall segments: basal, medial, and apical. Care was taken to obtain the best visualization of the right ventricle to enable accurate delineation of its endocardial border. Irregular cardiac cycles or those containing premature ventricular beats were excluded.

The intraobserver reproducibility of the strain measurements was assessed in a subsample of 30 randomly selected patients 3 months after the initial evaluation; the observer was blinded to the previous results. Interobserver variability was assessed in the same subsample by a second blinded experienced echocardiographer.

\section{Biomarkers}

Biomarker analysis was performed before EMB. For this, a $20 \mathrm{~mL}$ blood sample was collected from a peripheral veinto determine the plasma levels of cardiac troponin I and BNP.

Cardiac troponin I levels were quantified with a high sensitivity 3-step sandwich immunoassay using direct chemiluminescent technology and consistent amounts of 2 monoclonal antibodies. An auxiliary reagent was included to reduce nonspecific binding using an Advia Centaur TnI-Ultra commercial kit (Siemens Healthcare Diagnostics, Tarrytown, New York, USA). The level of detection was $0.006 \mathrm{ng} / \mathrm{mL}$ (levels $<0.006$ were reported as $0.005 \mathrm{ng} / \mathrm{mL}$ ). The normal range of cardiac troponin I was considered $<0.04 \mathrm{ng} / \mathrm{mL}$.

Plasma concentrations of BNP were determined with a 2-step sandwich immunoassay using direct 
chemiluminescent technology and consistent amounts of 2 monoclonal antibodies using an Advia Centaur commercial kit (Siemens Healthcare, Malvern, Pennsylvania, USA). The level of detection was $2 \mathrm{pg} / \mathrm{mL}$. Levels $<2$ were reported as $1 \mathrm{pg} / \mathrm{mL}$.

\section{Statistical analysis}

Categorical variables were compared using Pearson chisquare tests, Fisher exact tests, or likelihood ratio tests. Continuous variables were compared usingthe analysis of variance and Tukey's test (normal distribution) or the Kruskal-Wallis and Dunn's tests. The results were expressed as the means with standard deviations or as the medians with interquartile ranges. Linear correlations were tested using the Spearman rank method.

Receiver operating characteristic curves were constructed for variables significantly associated with the presence of ACR degree 2R, and the optimal cutoff values were calculated as the point with the highest sums of sensitivity and specificity. Receiver operating characteristic curves were also constructed for troponin combined with strain parameters significantly associated with the presence of ACR degree $2 \mathrm{R}$.

The interobserver and intraobserver reproducibility of LV-GLS, LV-Twist and RV-FWLSwere assessed using intraclass correlation coefficients and 95\% confidence intervals (CIs) in one-way random and two-way mixed models.

All analyses were performed using SPSS version 17 (SPSS Inc., Chicago, Illinois, USA). A $p$-value $<0.05$ was considered statistically significant.

\section{Results}

Ninety-five patients were initially enrolled in the study. Among these patients, 35 were excluded because of the following characteristics: 2 due to cardiac arrhythmia, 4 due to left ventricular systolic dysfunction, 4 due to right ventricular systolic dysfunction, 14 due to two or more previous ACR episodes, 4 due to a limited echocardiographic acoustic window for STE analysis, 3 due to inconclusive results of EMB, 2 due to humoral rejection and 2 due to Chagas disease recurrence. A total of 60

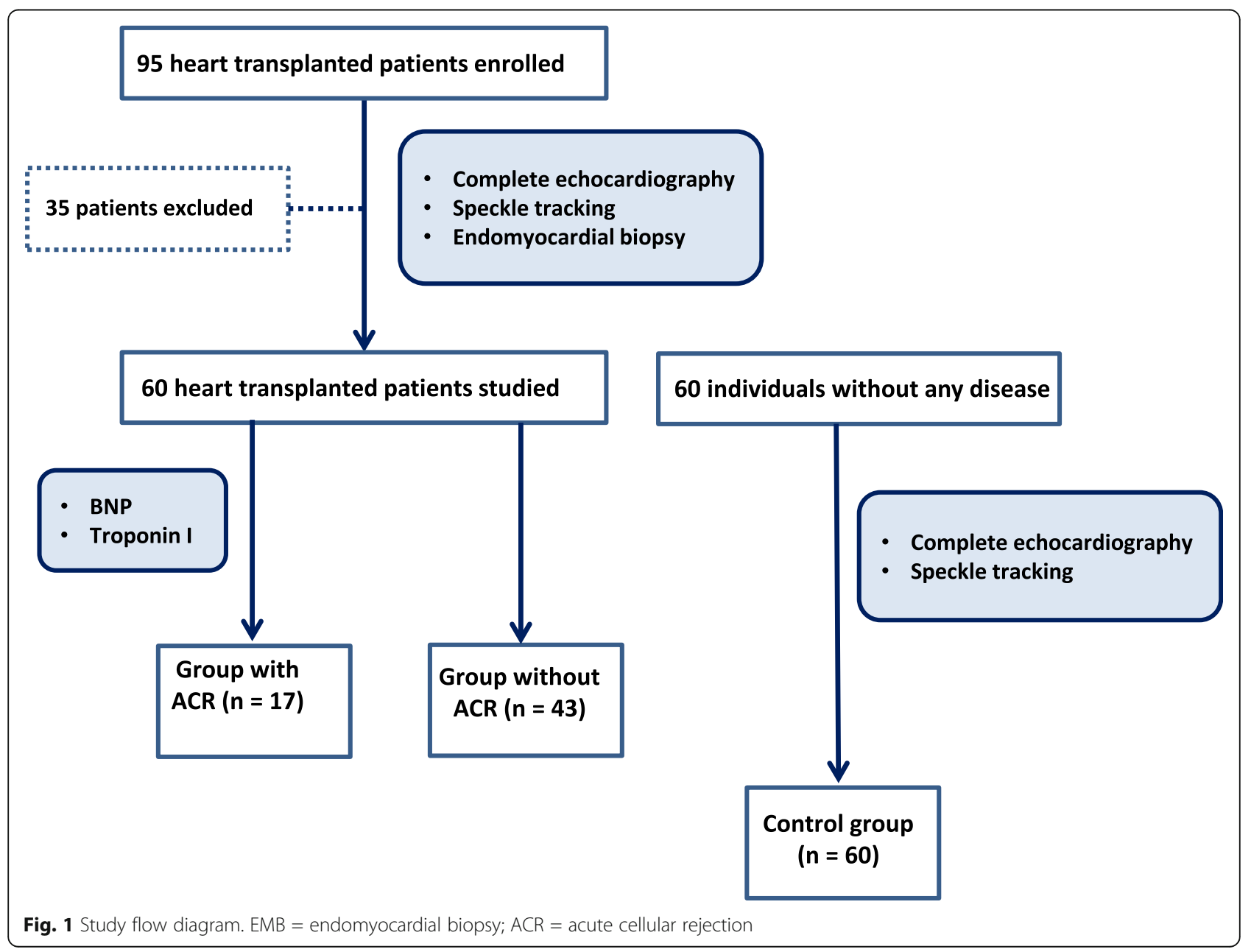


heart transplant patients and 60 control individuals constituted the final study population (Fig. 1).

We analyzed a total of 60 EMBs of heart transplant patients. All biopsies were performed at 6 months after heart transplantation. Among these 60 EMBs, 43 (72\%) did not show significant ACR (grade 0 or $1 \mathrm{R})$, while $17(28 \%)$ showed significant ACR (grade $2 R$ or $3 R$ ).

The baseline characteristics of the heart transplant patients included in this study are described in Table 1. The clinical characteristics of the groups with and without significant ACR detected by EMB, as well as those of the control group individuals, are described in Table 2.

Table 1 Baseline characteristics of the transplant patients

\begin{tabular}{ll}
\hline Characteristic & Heart transplant patients \\
& $\boldsymbol{n}=60$ \\
\hline Male sex & $34(56.7 \%)$ \\
Age (years) & $42.1 \pm 11.5$ \\
Weight (kg) & $61.3 \pm 13.4$ \\
Height (cm) & $162.5 \pm 10.3$ \\
Body surface area (m²) & $1.7 \pm 0.2$ \\
Primary disease & \\
Chagasic cardiomyopathy & $22(36.7 \%)$ \\
Idiopathic dilated cardiomyopathy & $21(35.0 \%)$ \\
Ischemic cardiomyopathy & $7(11.7 \%)$ \\
Valvular cardiomyopathy & $5(8.3 \%)$ \\
Hypertensive cardiomyopathy & $4(6.7 \%)$ \\
CTRCD & $1(1.6 \%)$ \\
Hypertension & $12(20.0 \%)$ \\
Diabetes & $12(20.0 \%)$ \\
Smoking & $17(28.3 \%)$ \\
Time since transplantation (months) & 6 \\
Medication &
\end{tabular}

The values are presented as the mean (standard deviation) or number (percentage)

CTRCD Cancer therapeutic-related cardiac dysfunction; $A C E I$ angiotensin converting enzyme inhibitor; $A R B$ aldosterone receptor blocker; $C C B$ calcium channel blocker

\section{Biomarkers}

Cardiac troponin I levels as assessed by the ultrasensitive assay were significantly higher in patients with significant ACR than in patients without significant ACR $[0.19 \mathrm{ng} / \mathrm{mL}(0.09-1.31)$ vs $0.05 \mathrm{ng} / \mathrm{mL}(0.01-0.18), p=$ 0.007]. No significant difference in BNP levels was found between patients with significant ACR and patients without significant ACR [264.5 pg/mL (160 to 976) vs 248 $\mathrm{pg} / \mathrm{mL}$ (90 to 528 ), $p=0.435$, respectively].

\section{Speckle tracking echocardiography}

The variables obtained by conventional echocardiography are shown in Table 3 . There were no differences between the group with significant ACR and the group without significant ACR regarding these parameters. However, heart transplant patients had a greater septum and inferolateral wall thickness, a larger left atrium diameter, a higher $E / E^{\prime}$, and higher relative thickness and left ventricular mass index values than control individuals. In addition, the heart transplant patients had a lower right ventricular fractional area change, systolic velocity of the tricuspid annulus, E' velocity, A' velocity and tricuspid annular plane systolic excursion than control individuals.

The LV-GLS, LV-GCS, LV-GRS, RV-FWLS and LVtwist values are shown in Table 4 . The absolute values of these variables were significantly lower in the heart transplant patients without rejection than in the control individuals, except for the LV-twist. In the group with significant ACR, the LV-GLS, LV-twist and RV-FWLS were significantly lower (in absolute values) than that in the groups without significant ACR $(12.5 \% \pm 2.9 \%$ vs $14.8 \% \pm 2.3 \%, p=0.002 ; 13.9^{\circ} \pm 4.8^{\circ}$ vs $17.1^{\circ} \pm 3.0^{\circ}, p=$ $0.048 ; 16.6 \% \pm 2.9 \% \mathrm{vs} 21.4 \% \pm 3.2 \%, p<0.001$; respectively), as shown in Fig. 2.

When we combined the strain parameters assessed as significant to detect ACR with troponin, we found that it increased the accuracy compared with the variables used individually. We report it in the model described below, based on the area under the curve (AUC) (Fig. 3).

The intraclass correlation coefficient of LV-GLS was $0.92(95 \% \mathrm{CI}=0.85-0.96)$ for interobserver variability. For intraobserver variability, the intraclass correlation coefficient of the LV-GLS was 0.93 (95\% CI $=0.86-$ 0.97). The intraclass correlation coefficients of the RVFWLS were $0.90(95 \% \mathrm{CI}=0.80-0.95)$ and $0.92(95 \% \mathrm{CI}$ $=0.84-0.96)$ for inter- and intraobserver variability, respectively. For the LV-Twist, the inter and intraobserver variability were $0.79(95 \% \mathrm{CI}=0.35-0.94)$ and $0.78(95 \%$ $\mathrm{CI}=0.33-0.94)$, respectively.

\section{Discussion}

The main results of our work are that patients with ACR had significantly lower values of LV-GLS, RV-FWLS and 
Table 2 Characteristics of the control group and groups with and without significant ACR

\begin{tabular}{|c|c|c|c|c|}
\hline Variable & $\begin{array}{l}\text { Control group } \\
(n=60)\end{array}$ & $\begin{array}{l}\text { Group without } \\
\text { significant ACR }(n=43)\end{array}$ & $\begin{array}{l}\text { Group with } \\
\text { significant ACR }(n=17)\end{array}$ & $p$ value \\
\hline Age (years) & $43 \pm 9$ & $44 \pm 11$ & $48 \pm 13$ & $0.20^{c}$ \\
\hline Male sex & $34(56.7 \%)$ & $24(55.8 \%)$ & $10(58.8 \%)$ & $0.139^{b}$ \\
\hline Weight (kg) & $72(63-80)$ & $70(58-81)$ & $71(61-87)$ & $0.684^{a}$ \\
\hline Height (cm) & $1.69 \pm 0.09$ & $1.62 \pm 0.09^{\#}$ & $1.59 \pm 0.13^{\#}$ & $<0.001^{c}$ \\
\hline $\mathrm{BMI}\left(\mathrm{kg} / \mathrm{m}^{2}\right)$ & $24.7(22.9-27.1)$ & $26.0(22.7-31.6)$ & $26.1(24.0-34.7)$ & $0.152^{a}$ \\
\hline $\mathrm{SBP}(\mathrm{mmHg})$ & $123 \pm 13$ & $126 \pm 19$ & $122 \pm 13$ & $0.672^{c}$ \\
\hline $\mathrm{DBP}(\mathrm{mmHg})$ & $76 \pm 10$ & $76 \pm 10$ & $72 \pm 8$ & $0.463^{c}$ \\
\hline PAP $(\mathrm{mmHg})$ & - & $30 \pm 10$ & $34 \pm 11$ & $0.465^{d}$ \\
\hline Hypertension & $0(0 \%)$ & 7 (16.3\%) & $5(29.4 \%)$ & $<0.001^{b}$ \\
\hline Diabetes mellitus & $0(0 \%)$ & 7 (16.3\%) & $5(29.4 \%)$ & $<0.001^{b}$ \\
\hline Smoking & $0(0 \%)$ & $14(32.6 \%)$ & $3(17.6 \%)$ & $<0.001^{b}$ \\
\hline $\mathrm{Cr}(\mathrm{mg} / \mathrm{dL})$ & $0.80(0.5-1.0)$ & $1.19(0.9-1.34)$ & $1.1(0.95-1.4)$ & $0.930^{a}$ \\
\hline $\mathrm{BSA}\left(\mathrm{m}^{2}\right)$ & $1.60(1.55-1.68)$ & $1.78(1.58-1.93)^{\#}$ & $1.75(1.60-2.03)^{\#}$ & $<0.001^{a}$ \\
\hline
\end{tabular}

The values are presented as the mean (standard deviation), median (interquartile range) or number (percentage). $B M I$ body mass index; $C r$ creatinine; $B S A$ body surface area; SBP Systolic blood pressure; DBP diastolic blood pressure; PAP pulmonary artery pressure; $A C R$ acute cellular rejection ${ }^{\mathrm{a}}$ Kruskal-Wallis test; ${ }^{\mathrm{b}}$ Pearson chi-square; ${ }^{\mathrm{C}}$ ANOVA

$\# p<0.05$ (vs. the control group)

Table 3 Echocardiographic parameters and their relationships with significant acute cellular rejection

\begin{tabular}{|c|c|c|c|c|}
\hline Variable & $\begin{array}{l}\text { Control } \\
\text { group }\end{array}$ & $\begin{array}{l}\text { Group without } \\
\text { ACR }\end{array}$ & $\begin{array}{l}\text { Group with } \\
\text { ACR }\end{array}$ & $p$ value \\
\hline Left atrium (mm) & $34(31-36)$ & 41 (38-46)\# & $43(35-49) \#$ & $<0.001^{\mathrm{a}}$ \\
\hline LA Volume $\left(\mathrm{mm}^{3} / \mathrm{cm}^{2}\right)$ & $25.02 \pm 2.97$ & $33.31 \pm 4.96 \#$ & $33.78 \pm 3.06 \#$ & $<0.001^{b}$ \\
\hline Septum (mm) & $8(8-9)$ & $11(10-13) \#$ & $12(10-13) \#$ & $<0.001^{\mathrm{a}}$ \\
\hline Inferolateral wall (mm) & $8(8-9)$ & $11(10-11) \#$ & 11 (9-13)\# & $<0.001^{a}$ \\
\hline Relative thickness & $0.38(0.35-0.41)$ & $0.49(0.44-0.53) \#$ & $0.47(0.40-0.55) \#$ & $<0.001^{\mathrm{a}}$ \\
\hline $\operatorname{LVMI}\left(\mathrm{g} / \mathrm{m}^{2}\right)$ & $71(65-83)$ & 102 (86-124)\# & 109 (80-141)\# & $<0.001^{a}$ \\
\hline LVEF (\%) & $65(62-68)$ & $64(62-67)$ & $65(63-67)$ & $0.739^{a}$ \\
\hline E velocity (m/s) & $0.84(0.69-0.93)$ & $0.65(0.60-0.76) \#$ & $0.66(0.54-0.84) \#$ & $<0.001^{\mathrm{a}}$ \\
\hline DT (ms) & 189 (168-208) & $187(144-214)$ & $190(153-207)$ & $0.787^{a}$ \\
\hline A velocity (m/s) & $0.50(0.42-0.58)$ & $0.41(0.32-0.50) \#$ & $0.42(0.38-0.68) \#$ & $0.001^{\mathrm{a}}$ \\
\hline$E / A$ & $1.60(1.38-1.93)$ & $1.64(1.29-2.00)$ & $1.46(1.29-1.75)$ & $0.543^{a}$ \\
\hline $\mathrm{S}^{\prime}$ velocity $(\mathrm{m} / \mathrm{s})$ & $0.08(0.07-0.09)$ & $0.07(0.06-0.08) \#$ & $0.07(0.05-0.08)$ & $0.010^{\mathrm{a}}$ \\
\hline$E^{\prime}$ velocity $(\mathrm{m} / \mathrm{s})$ & $0.11(0.09-0.12)$ & $0.06(0.05-0.08) \#$ & $0.07(0.05-0.07) \#$ & $<0.001^{\mathrm{a}}$ \\
\hline$A^{\prime}$ velocity (m/s) & $0.08(0.07-0.10)$ & $0.06(0.05-0.08) \#$ & $0.08(0.06-0.09) \#$ & $<0.001^{a}$ \\
\hline$E / E^{\prime}$ & $7.92(6.63-8.78)$ & 10.14 (7.99-12.96)\# & 10 (7.93-14.21)\# & $<0.001^{\mathrm{a}}$ \\
\hline FAC (\%) & $43(40-48)$ & $39(36-45) \#$ & $39(37-42) \#$ & $0.001^{\mathrm{a}}$ \\
\hline TAPSE $(\mathrm{mm})$ & $24(20-26)$ & $17(15-18) \#$ & $16(15-17) \#$ & $<0.001^{\mathrm{a}}$ \\
\hline $\mathrm{S}^{\prime}$ velocity $(\mathrm{m} / \mathrm{s})$ of tricuspid annulus & $13(12-15)$ & $12(11-14)$ & $11(11-12) \#$ & $<0.001^{\mathrm{a}}$ \\
\hline
\end{tabular}

The values are presented as the mean (standard deviation) or median (interquartile range)

${ }^{a}$ Kruskal-Wallis test; $\# p<0.05$ (vs. the control group). ACR acute cellular rejection; $L A$ left atrium; DT deceleration time; $L V E F$ left ventricular ejection fraction; FAC fractional area change; lat lateral; LVMI left ventricular mass index; TAPSE tricuspid annular plane systolic excursion 
Table 4 LV-twist, LV-GLS, LV-GCS, LV-GRS and RV-FWLS values and their relationships with cardiac rejection in univariate analysis

\begin{tabular}{|c|c|c|c|c|c|c|c|}
\hline Variable & $\begin{array}{l}\text { Control } \\
\text { group } \\
(n=60)\end{array}$ & $\begin{array}{l}\text { Without } \\
\text { ACR } \\
(n=43)\end{array}$ & $\begin{array}{l}\text { With } \\
\text { ACR } \\
(n=17)\end{array}$ & $P^{t}$ & $P^{\neq}$ & $P^{*}$ & Overall \\
\hline LV-twist $\left({ }^{\circ}\right)$ & $18.8 \pm 4.63$ & $17.1 \pm 3.02$ & $13.9 \pm 4.79$ & 0.402 & 0.002 & 0.048 & 0.001 \\
\hline LV-GLS (\%) ${ }^{A V}$ & $21.2 \pm 2.1$ & $14.8 \pm 2.3$ & $12.5 \pm 2.9$ & $<0.001$ & $<0.001$ & 0.002 & $<0.001$ \\
\hline LV-GCS $(\%)^{A V}$ & $21.3 \pm 3.0$ & $16.2 \pm 4.3$ & $15.2 \pm 2.8$ & $<0.001$ & $<0.001$ & 0.574 & $<0.001$ \\
\hline LV-GRS (\%) & $41.2 \pm 12.9$ & $28.6 \pm 9.9$ & $28.3 \pm 8.8$ & $<0.000$ & $<0.001$ & 0.996 & $<0.001$ \\
\hline RV-FWLS (\%) ${ }^{\mathrm{AV}}$ & $25.0 \pm 3.3$ & $21.4 \pm 3.2$ & $16.6 \pm 2.9$ & $<0.000$ & $<0.001$ & $<0.001$ & $<0.001$ \\
\hline
\end{tabular}

$A V$ absolute value; $A C R$ acute cellular rejection

Data are expressed as the mean \pm SD

† Control group vs group without significant ACR

$\neq$ Control group vs group with significant ACR

*Group without ACR vs group with significant $A C R$

$\S$ Analysis of variance global $P$ value between groups

LV-Twist and higher level of troponin I than patients without significant ACR. In addition, the combination of troponin and these parameters may improve the diagnostic value of strain in heart transplant patients with preserved left and right ventricular systolic functions. The combination of RV-FWLS and troponin presented a higher accuracy for the detection of ACR degree $\geq 2 \mathrm{R}$. The AUC of the combination of troponin $(>0.05 \mathrm{ng} / \mathrm{mL})$ with RV-FWLS (with a cut-off value <18\%) was $0.89(95 \% \mathrm{CI}=0.81-0.93)$. Thus, it may be considered for use as a screening tool for the detection of ACR and could reduce the number of EMBs after heart transplantation.
ACR is a significant and frequent complication of heart transplantation. In the first year, it is the most common cause of mortality. Currently, EMB is the clinical gold standard in screening for graft rejection after heart transplantation and is actually the only tool for the diagnosis and classification of allograft rejection [5]. Considerable efforts have been made to improve the consistency, reliability and reproducibility of the histopathological evaluation of EMB. However, several issues make EMB assessment more difficult and less reproducible than it should be. Critical issues include the subjective and challenging pathological interpretation of EMBs and the risks associated with the procedure [25].

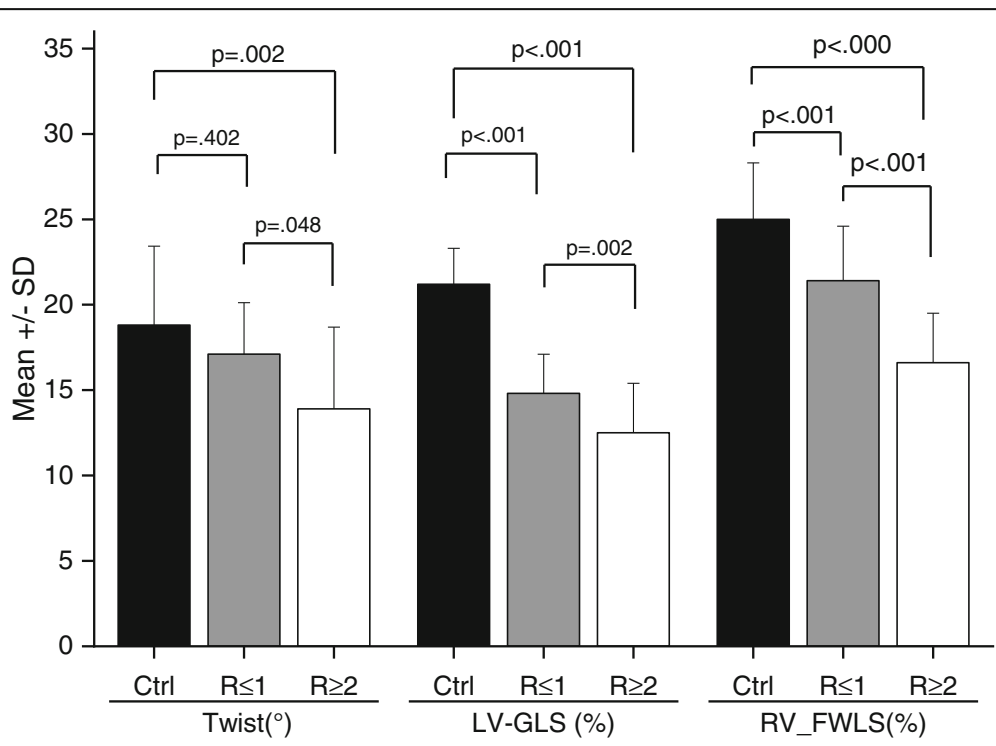

Fig. 2 Twist, left ventricular global longitudinal strain and right ventricular free wall longitudinal strain in the control group and in two groups of heart transplant patients, those with and those without significant acute cellular rejection $(R \geq 2$ vs. $R \leq 1)$ ANOVA: F-test. *Significative to $5 \%$ (Tukey test for all pairwise comparisons); 1: Ctrl = Control (Cardiac untransplanted patients); 2 : Heart transplant patients without rejection (R $\leq 1$ ); 3: Heart transplant patients with rejection $(R \geq 2)$. SD: Standard Deviation; LV-GLS: Left Ventricular Global Longitudinal Strain; RV-FWLS, Right Ventricular Free Wall Longitudinal Strain 


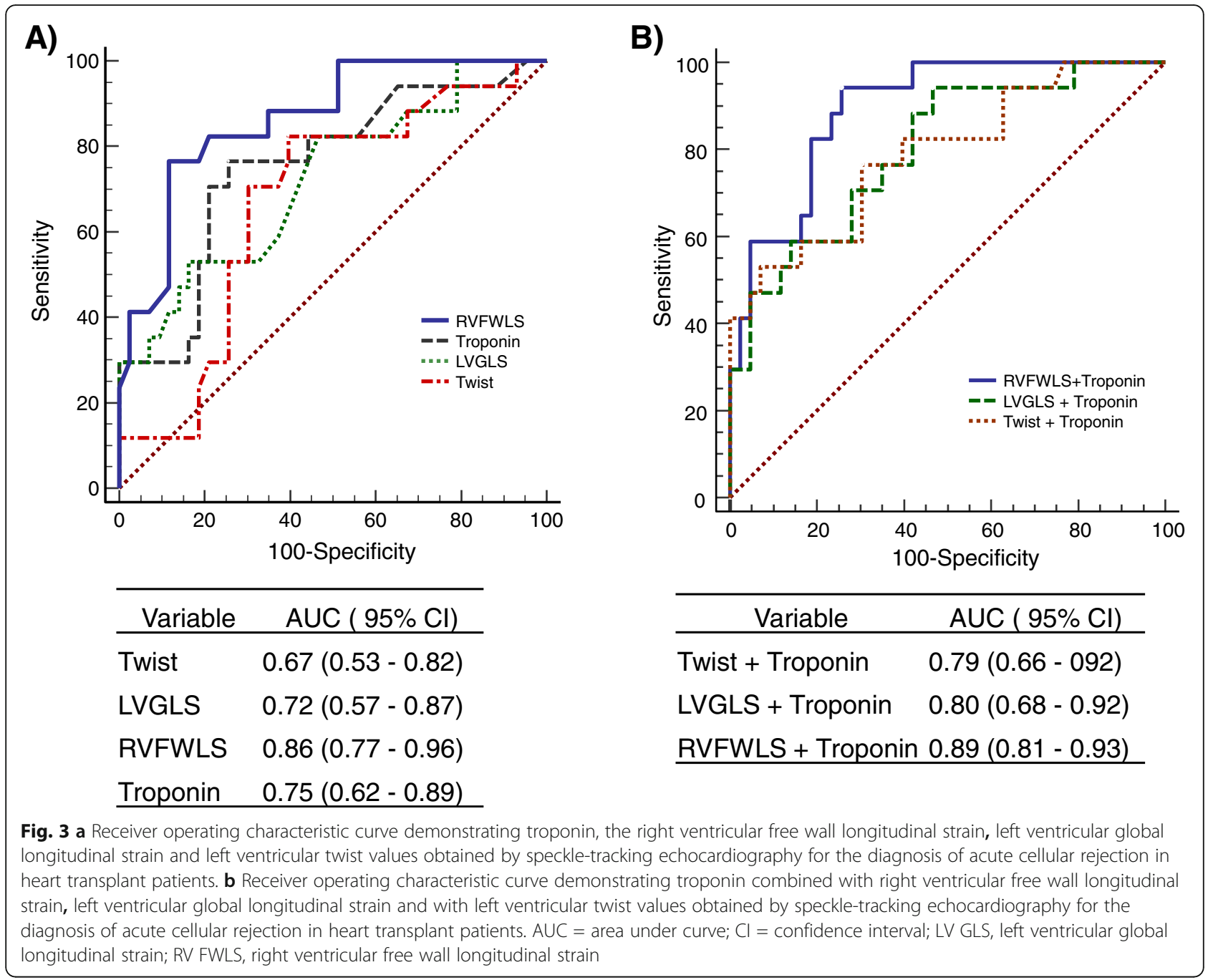

Considering these limitations, noninvasive techniques to detect cardiac rejection have been evaluated $[14,26]$.

To the best of our knowledge, this is the first study to analyze all strain parameters using STE (LV-GLS, LVGRS, LV-GCS, LV-Twist and RV-FWLS) in the same population of heart transplanted patients with normal right and left ventricular systolic function, and its combination with biomarkers, to diagnosis clinically significant ACR. Extra care was given in our study to restrict patient selection to a fixed period of time $(6$ months post-heart transplantation) to minimize the possible influence of the time since heart transplantation on ventricular strain, as recommended by the current guidelines [27]. Additionally, this fixed period of selection minimized the possible bias of pre transplantation ischemic injury, which can manifest up to the sixth month post heart transplantation. The first 6 months is a period of adaptation, during which many patients can still present some degree of right ventricular systolic dysfunction.
Previous studies have shown that myocardial strain has a higher sensitivity than conventional echocardiography, and therefore, may be an important tool to detect early subclinical cardiac dysfunction [28]. Although myocardial strain imaging has been reported to have potential for the detection of graft dysfunction in the early stage, its diagnostic value has not been widely recognized yet $[19,20]$. Similarly, as reported in a recent meta-analysis, our study showed that the LV-GLS was lower in transplant patients with significant ACR compared with patients without significant ACR [29]. This may occur secondary to myocardial deformation, and it may be impaired due to inflammatory cellular infiltration and myocardial edema, and can be reflected by myocardial strain parameters. However, the diagnostic value of other strain parameters by 2D STE on ACR detection is still controversial.

Right ventricular strain analysis has been poorly described to date in this scenario. Mingo-Santos et al. demonstrated a predictive role of STE parameters in the 
diagnosis of ACR (RV-FWLS and LV-GLS with threshold values of $<17$ and $<15.5 \%$, respectively) [30]. That study classified biopsies into 3 groups $(0,1 R$, and $\geq 2 R)$. However, our study divided the biopsy results into two groups according to the grade of rejection: biopsies without significant rejection ( 0 and $1 \mathrm{R})$ and biopsies with significant rejection $(\geq 2 \mathrm{R})$. This division was based upon the clinical meaning of the rejection grade, since cases with grades of 0 or $1 \mathrm{R}$ do not require an immediate intervention via adjustment of immunosuppressive medications, whereas this adjustment is necessary in patients presenting with $2 \mathrm{R}$ and $3 \mathrm{R}$ rejection. In agreement with the study from Mingo-Santos et al. [30],our study confirms the reduction in left ventricular and right ventricular STE parameters during ACR $\geq 2$. Our cutoff value was slightly higher than that reported by MingoSantos et al. [30]. We speculate that it might be due to the use of different echocardiographic equipment or even the characteristics of the studied populations. Unfortunately, the investigators did not analyze LV-Twist and troponin.

As we showed in our results, the LV-Twist values were significantly lower in the group with significant ACR than in the group without significant ACR $\left(13.9^{\circ} \pm 4.79^{\circ}\right.$ vs $17.1^{\circ} \pm 3.02^{\circ}, p<0.048$ ). In parallel to our results, a unique previous study that applied STE derived LVTwist measurements to detect rejection in heart transplanted patients demonstrated that the LV-Twist decreased more in the group with ACR than in the group without ACR $\left(9.6^{\circ} \pm 2.7^{\circ}\right.$ vs $\left.12.2^{\circ} \pm 2.3^{\circ}\right), p<0.0001$, [31]. We postulated that twist preceded the deterioration in left ventricular ejection fraction, suggesting early myocardial involvement in cardiac rejection. With the advances of technology that have made this technique more available and increasingly feasible, this parameter of cardiac mechanics has been increasingly studied in other pathological situations, and can be applied in this type of patient [32, 33].

As acute rejection promotes cardiomyocyte necrosis and results in compromised cardiac mechanics, troponin and BNP have been evaluated as potential diagnostic tools for ACR [34, 35]. There have been controversial results on these biomarkers in the field of heart transplantation [15, 36]. Our study used an ultrasensitive assay for cardiac troponin I that detects 10 to 100 times lower levels than standard assays. Troponin was measured before the biopsy, so this procedure did not interfere with its serum levels. Troponin I levels were significantly elevated in patients with significant ACR. Serum BNP levels were not different between the groups; this finding can be explained by the suggestion of some studies that BNP remains altered in most patients for up to 1 year after heart transplantation [37, 38]. In accordance with Bader et al. [39], we also observed that BNP levels did not predict rejection after heart transplantation, and we suggest that BNP is not clinically useful for the detection of ACR.

In accordance with previous reports in the literature [40], we confirmed in our population that heart transplant patients have a characteristic cardiac geometric remodeling, featured by a greater septum and inferolateral wall thickness, a larger left atrium diameter, and greater left ventricular mass index values than matched non transplanted controls. Heart transplant patients also showed lower values for conventional right ventricular systolic function parameters, such as fractional area change, tricuspid annulus systolic velocity and tricuspid annular plane systolic excursion. Moreover, regarding left ventricular diastolic function, heart transplant patients had lower tissue Doppler velocities and higher $\mathrm{E} / \mathrm{e}^{\prime}$ ratios, suggesting impaired relaxation and increased left ventricular filling pressures.

This study demonstrated that heart transplant patients without rejection present unique ventricular dynamics, characterized by lower LV-GLS, LV-GCS, LV-GRS and RV-FWLS, in comparison with control individuals. We have confirmed the data recently published by Ingvarsson et al. [40], which showed that echocardiographic measurements from 124 heart transplant patients were different from the reference values except for LV-GCS. Unfortunately, the investigators did not analyze LVTwist. In our study, we used a non-transplanted control group matched by age and sex to confirm these results. Multiple mechanisms may explain the different echocardiographic findings in heart transplant patients. Their pathophysiology involves the consequences of surgical trauma, such as ischemic injury and the release of inflammatory mediators, in addition to previous pulmonary hypertension compromising right ventricular dynamics and the risks associated with rejection, cardiac biopsies and immunosuppressive medications.

Our data failed to find any association between diastolic markers and rejection. The results found in the literature are highly conflicting and could not be reproduced by our data. This can be explained by a limitation of diastolic dysfunction parameters due to their dependence on heart rate (which is generally elevated in transplanted patients, with a fusion of $\mathrm{E}$ and $\mathrm{A}$ waves), loading conditions and donor age [41-45].

\section{Limitations}

The limitations of this study should be addressed. First, this was a single-center study with a small number of patients and a limited number of rejection episodes graded equal to or above 2R (17 out of 60 samples). Despite its extensive validation, STE is still an evolving technique, and improvements such as better tracking accuracy are still needed. Additionally, STE accuracy is highly dependent on image quality. Suboptimal resolution can produce a negative impact 
on the final results. Nevertheless, despite these limitations, we were able to successfully perform speckletracking analysis of both left and right ventricular longitudinal strain in $95 \%$ of the patients. The reproducibility of the parameters was good and was similar to that reported in other studies. Finally, our results must be independently validated in a prospective external cohort, preferably in multicenter studies, before they can be used in clinical practice.

\section{Conclusions}

Heart transplant patients have altered left ventricular dynamics compared with control individuals. The association of strain parameters derived from STE, particularly RV-FWLS, with troponin seems to be able to detect ACR with higher accuracy than these variables used individually. These parameters might be considered a screening method that can be added to the management of heart transplant patients to safely reduce the number of unnecessary EMB.

\section{Perspectives}

With the increase in heart transplantation, new tools for detecting acute cellular rejection are highly desirable. Our study findings show that the analysis of strain parameters with troponin levels might be useful to noninvasively detect patients who are at high risk of cellular rejection.

\section{Acknowledgements}

Not applicable.

\section{Authors' contributions}

All authors contributed to the manuscript: Wilson Mathias Jr. and Jeane M. Tsutsui conducted the study conceptualization, writing and review of the manuscript, Cecilia B.B.V. Cruz worked with data acquisition, analysis and writing, Ludhmila A. Hajjar and Fernando Bacal worked with clinical data acquisition of the patients, Marco L. Alves, Márcio S.M. Lima and Maria C. Abduch performed data analysis and curation, Marcelo L.C. Viera and Hsu P. Chiang performed review and editing of the manuscript, Juliana B. C. Salviano performed project administration and writing, Julia T Fukushima made the statistical analysis, Joao C.N. Sbano and Isabela B.S.C participated in the acquisition of data performed data acquisition, writing and reviewing of the manuscript. All authors have read and approved the final version of the manuscript.

\section{Authors' information}

Not applicable.

\section{Funding}

Not applicable.

\section{Availability of data and materials}

All data generated or analysed during this study are included in this published article. If you have questions or additional information, the datasets used and/or analysed during the current study are available from the corresponding author upon reasonable request.

\section{Ethics approval and consent to participate}

This article has been approved by the Ethics and Research Committee of the Heart Institute University of Sao Paulo.

\section{Consent for publication}

All authors listed on the title page have read the manuscript, attest to the validity and legitimacy of the data and its interpretation, and agree to its submission to Cardiovascular Ultrasound.

\section{Competing interests}

The authors declare that they have no competing interests.

Received: 19 October 2020 Accepted: 21 December 2020

Published online: 09 January 2021

\section{References}

1. Luckraz H, Sharples LD, Charman SC, Tsui SS, Wallwork J, Parameshwar J, et al. Does heart transplantation confer survival benefit in all risk groups? J Heart Lung Transplant. 2005;24(9):1231-4.

2. Frazier $\mathrm{OH}$. Current status of cardiac transplantation and left ventricular assist devices. Tex Heart Inst J. 2010;37(3):319-21.

3. Patel JK, Kittleson M, Kobashigawa JA. Cardiac allograft rejection. Surgeon. 2011;9(3):160-7.

4. Boyle A. Current status of cardiac transplantation and mechanical circulatory support. Curr Heart Fail Rep. 2009;6(1):28-33.

5. Murphy J, Frantz R, Cooper L. Endomyocardial biopsy (123). In: Murphy J, Lloyd M, editors. Mayo Clinic Cardiology Concise Textbook. Minnesota: Rochester; 2007. p. 1481

6. Saraiva F, Matos V, Goncalves L, Antunes M, Providencia LA. Complications of endomyocardial biopsy in heart transplant patients: a retrospective study of 2117 consecutive procedures. Transplant Proc. 2011;43(5):1908-12.

7. Yilmaz A, Kindermann I, Kindermann M, Mahfoud F, Ukena C, Athanasiadis A, et al. Comparative evaluation of left and right ventricular endomyocardial biopsy: differences in complication rate and diagnostic performance. Circulation. 2010;122(9):900-9.

8. Holzmann M, Nicko A, Kuhl U, Noutsias M, Poller W, Hoffmann W, et al. Complication rate of right ventricular endomyocardial biopsy via the femoral approach: a retrospective and prospective study analyzing 3048 diagnostic procedures over an 11-year period. Circulation. 2008;118(17): 1722-8.

9. Deckers JW, Hare JM, Baughman KL. Complications of transvenous right ventricular endomyocardial biopsy in adult patients with cardiomyopathy: a seven-year survey of 546 consecutive diagnostic procedures in a tertiary referral center. J Am Coll Cardiol. 1992;19(1):43-7.

10. Zakliczynski M, Nozynski J, Konecka-Mrowka D, Pyka L, Trybunia D, Swierad $M$, et al. Quilty effect correlates with biopsy-proven acute cellular rejection but does not predict transplanted heart coronary artery vasculopathy. J Heart Lung Transplant. 2009;28(3):255-9.

11. Pardo-Mindan FJ, Lozano MD. "Quilty effect" in heart transplantation: is it related to acute rejection? J Heart Lung Transplant. 1991;10(6):937-41.

12. Forbes RD, Rowan RA, Billingham ME. Endocardial infiltrates in human heart transplants: a serial biopsy analysis comparing four immunosuppression protocols. Hum Pathol. 1990;21(8):850-5.

13. Wu AH, Feng YJ, Moore R, Apple FS, McPherson PH, Buechler KF, et al. Characterization of cardiac troponin subunit release into serum after acute myocardial infarction and comparison of assays for troponin $\mathrm{T}$ and $\mathrm{I}$. American association for clinical chemistry subcommittee on cTnl standardization. Clin Chem. 1998:44(6 Pt 1):1198-208.

14. Holweg CT, Potena L, Luikart H, Yu T, Berry GJ, Cooke JP, et al. Identification and classification of acute cardiac rejection by intragraft transcriptional profiling. Circulation. 2011;123(20):2236-43.

15. Fitzsimons S, Evans J, Parameshwar J, Pettit SJ. Utility of troponin assays for exclusion of acute cellular rejection after heart transplantation: a systematic review. J Heart Lung Transplant. 2018;37(5):631-8.

16. Garrido IP, Pascual-Figal DA, Nicolas F, Gonzalez-Carrillo MJ, ManzanoFernandez S, Sanchez-Mas J, et al. Usefulness of serial monitoring of B-type natriuretic peptide for the detection of acute rejection after heart transplantation. Am J Cardiol. 2009;103(8):1149-53.

17. Bansal M, Kasliwal RR. How do I do it? Speckle-tracking echocardiography. Indian Heart J. 2013;65(1):117-23.

18. Collier $P$, Phelan $D$, Klein A. A test in context: myocardial strain measured by speckle-tracking echocardiography. J Am Coll Cardiol. 2017;69(8):1043-56.

19. Tseng AS, Gorsi US, Barros-Gomes S, Miller FA, Pellikka PA, Clavell AL, et al. Use of speckle-tracking echocardiography-derived strain and systolic strain 
rate measurements to predict rejection in transplant hearts with preserved ejection fraction. BMC Cardiovasc Disord. 2018;18(1):241.

20. Sera F, Kato TS, Farr M, Russo C, Jin Z, Marboe CC, et al. Left ventricular longitudinal strain by speckle-tracking echocardiography is associated with treatment-requiring cardiac allograft rejection. J Card Fail. 2014;20(5):359-64.

21. Stewart S, Winters GL, Fishbein MC, Tazelaar HD, Kobashigawa J, Abrams J, et al. Revision of the 1990 working formulation for the standardization of nomenclature in the diagnosis of heart rejection. J Heart Lung Transplant. 2005;24(11):1710-20

22. Lang RM, Badano LP, Mor-Avi V, Afilalo J, Armstrong A, Ernande L, et al. Recommendations for cardiac chamber quantification by echocardiography in adults: an update from the American Society of Echocardiography and the European Association of Cardiovascular Imaging. Eur Heart J Cardiovasc Imaging. 2015;16(3):233-70.

23. Devereux RB, Alonso DR, Lutas EM, Gottlieb GJ, Campo E, Sachs I, et al. Echocardiographic assessment of left ventricular hypertrophy: comparison to necropsy findings. Am J Cardiol. 1986;57(6):450-8.

24. Mor-Avi V, Lang RM, Badano LP, Belohlavek M, Cardim NM, Derumeaux G, et al. Current and evolving echocardiographic techniques for the quantitative evaluation of cardiac mechanics: ASE/EAE consensus statement on methodology and indications endorsed by the Japanese Society of Echocardiography. Eur J Echocardiogr. 2011;12(3):167-205.

25. Strecker T, Rosch J, Weyand M, Agaimy A. Endomyocardial biopsy for monitoring heart transplant patients: 11-years-experience at a german heart center. Int J Clin Exp Pathol. 2013;6(1):55-65.

26. Wu YL, Ye Q, Ho C. Cellular and functional imaging of cardiac transplant rejection. Curr Cardiovasc Imaging Rep. 2011;4(1):50-62.

27. Badano LP, Miglioranza MH, Edvardsen T, Colafranceschi AS, Muraru D, Bacal F, et al. European Association of Cardiovascular Imaging/cardiovascular imaging Department of the Brazilian Society of cardiology recommendations for the use of cardiac imaging to assess and follow patients after heart transplantation. Eur Heart J Cardiovasc Imaging. 2015; 16(9):919-48.

28. Geyer H, Caracciolo G, Abe H, Wilansky S, Carerj S, Gentile F, et al. Assessment of myocardial mechanics using speckle tracking echocardiography: fundamentals and clinical applications. J Am Soc Echocardiogr. 2010;23(4):351-69 quiz 453-5.

29. Elkaryoni A, Altibi AM, Khan MS, Okasha O, Ellakany K, Hassan A, et al. Global longitudinal strain assessment of the left ventricle by speckle tracking echocardiography detects acute cellular rejection in orthotopic heart transplant recipients: A systematic review and meta-analysis. Echocardiography (Mount Kisco, NY). 2020;37(2):302-9.

30. Mingo-Santos S, Monivas-Palomero V, Garcia-Lunar I, Mitroi CD, GoirigolzarriArtaza J, Rivero B, et al. Usefulness of two-dimensional strain parameters to diagnose acute rejection after heart transplantation. J Am Soc Echocardiogr. 2015;28(10):1149-56

31. Sato T, Kato TS, Komamura K, Hashimoto S, Shishido T, Mano A, et al. Utility of left ventricular systolic torsion derived from 2-dimensional speckletracking echocardiography in monitoring acute cellular rejection in heart transplant recipients. J Heart Lung Transplant. 2011;30(5):536-43.

32. Zhang Y, Li SY. Xie JJ. Clin Cardiol: Wu Y. Twist/untwist parameters are promising evaluators of myocardial mechanic changes in heart failure patients with preserved ejection fraction; 2020.

33. Jia FW, Hsu J, Liu XH, Feng XJ, Pang HY, Lin X, et al. Usefulness of myocardial strain and twist for early detection of myocardial dysfunction in patients with autoimmune diseases. Am J Cardiol. 2020;125(3):475-81.

34. Zimmermann R, Baki S, Dengler TJ, Ring GH, Remppis A, Lange R, et al. Troponin T release after heart transplantation. Br Heart J. 1993;69(5):395-8.

35. Dengler TJ, Zimmermann R, Braun K, Muller-Bardorff M, Zehelein J, Sack FU, et al. Elevated serum concentrations of cardiac troponin $T$ in acute allograft rejection after human heart transplantation. J Am Coll Cardiol. 1998;32(2): 405-12.

36. Talha S, Charloux A, Piquard F, Geny B. Brain natriuretic peptide and right heart dysfunction after heart transplantation. Clin Transplant. 2017;31(6). https://doi.org/10.1111/ctr.12969.

37. Hervas I, Almenar L, Perez-Pastor JL, Chirivella M, Osa A, Martinez-Dolz L, et al. Radioimmunometric assay of B-type natriuretic peptide (BNP) in heart transplantation: correlation between BNP determinations and biopsy grading of rejection. Nucl Med Commun. 2003;24(8):925-31.
38. Gardner RS, Chong KS, Murday AJ, Morton JJ, McDonagh TA. N-terminal brain natriuretic peptide is predictive of death after cardiac transplantation. Heart. 2006;92(1):121-3.

39. Bader FM, Rogers RK, Kfoury AG, Gilbert EM, Horne BD, Stehlik J, et al. Timedependent changes in B-type natriuretic peptide after heart transplantation: correlation with allograft rejection and function. Congest Heart Fail. 2009; 15(2):63-7.

40. Ingvarsson A, Evaldsson AW, Waktare J, Nilsson J, Smith GJ, Stagmo M, et al. Normal reference ranges for transthoracic echocardiography following heart transplantation. Journal of the American Society of Echocardiography. 2018; 31(3):349-60.

41. Mena C, Wencker D, Krumholz HM, McNamara RL. Detection of heart transplant rejection in adults by echocardiographic diastolic indices: a systematic review of the literature. J Am Soc Echocardiogr. 2006;19(10): 1295-300.

42. Dandel M, Hummel M, Muller J, Wellnhofer E, Meyer R, Solowjowa N, et al. Reliability of tissue Doppler wall motion monitoring after heart transplantation for replacement of invasive routine screenings by optimally timed cardiac biopsies and catheterizations. Circulation. 2001;104(12 Suppl 1):1184-91.

43. Sun JP, Abdalla IA, Asher CR, Greenberg NL, Popovic ZB, Taylor DO, et al. Non-invasive evaluation of orthotopic heart transplant rejection by echocardiography. J Heart Lung Transplant. 2005;24(2):160-5.

44. Sade LE, Sezgin A, Ulucam M, Taymaz S, Simsek V, Tayfun E, et al. Evaluation of the potential role of echocardiography in the detection of allograft rejection in heart transplant recipients. Transplant Proc. 2006;38(2):636-8.

45. Stengel SM, Allemann Y, Zimmerli M, Lipp E, Kucher N, Mohacsi P, et al. Doppler tissue imaging for assessing left ventricular diastolic dysfunction in heart transplant rejection. Heart. 2001;86(4):432-7.

\section{Publisher's Note}

Springer Nature remains neutral with regard to jurisdictional claims in published maps and institutional affiliations.

Ready to submit your research? Choose BMC and benefit from:

- fast, convenient online submission

- thorough peer review by experienced researchers in your field

- rapid publication on acceptance

- support for research data, including large and complex data types

- gold Open Access which fosters wider collaboration and increased citations

- maximum visibility for your research: over $100 \mathrm{M}$ website views per year

At $\mathrm{BMC}$, research is always in progress.

Learn more biomedcentral.com/submissions 